\title{
Full title: A choice experiment to identify the most important elements of a successful cancer screening programme according to those who research and manage such programmes
}

Short title: Choice experiment for effective cancer screening

Jennifer Priaulx MPH ${ }^{1^{*}}$, Marcell Csanádi MSc² , Prof Harry J. de Koning, MD PhD ${ }^{3}$, Prof Martin McKee, CBE MD DSc FMedSci ${ }^{1}$

Corresponding author: Jennifer Priaulx, London School of Hygiene and Tropical Medicine, 15-17 Tavistock Place, London, WC1H 9SH, UK

E-mail: Jennifer.priaulx@Ishtm.ac.uk

${ }^{1}$ London School of Hygiene and Tropical Medicine, London, UK

${ }^{2}$ Syreon Research Institute, Budapest, Hungary

${ }^{3}$ Erasmus MC, University Medical Center Rotterdam, Department of Public Health, Rotterdam, The Netherlands

Acknowledgements

We would like to thank the support of those working on the EU-TOPIA project.

Funding statement

This study is part of the EU-TOPIA project funded by the EU-Framework Program (Horizon 2020) of the European Commission, project reference 634753; PI: prof HJ de Koning, MD $\mathrm{PhD}$, Erasmus MC.

Declarations

There are no declarations of conflicting interest 


\section{Abstract}

\section{Purpose}

The relationship between cancer screening activities in Europe and the health systems in which they are embedded varies, with some screening programmes organised largely separately, and others using existing health service staff and facilities. Whatever the precise arrangements, the opportunity for screening to achieve health gain depends on many elements interacting within and beyond the health system, from an accurate register identifying the target population to a means to ensure and monitor follow up.

\section{Method}

A conjoint analysis was undertaken with 66 cancer screening experts from 31 countries taking part in EU-TOPIA (Towards improved screening for breast, cervical and colorectal cancer in all of Europe) to identify priorities for an effective screening programme, taking a whole system perspective. Ten attributes, each with two levels, were derived from a review of the literature and consultation with experts in cancer screening. Statistical software generated 12 profiles that were ranked by respondents and analysed using standard conjoint analysis.

\section{Findings and conclusion}

The most important attributes were having up-to-date and evidence-based guidelines, followed by mechanisms for systematic monitoring of screening uptake, having a population register covering all of the eligible population and monitoring long-term outcomes. In discussions about the results, participants argued that quality assurance and adherence to guidelines were important, even though they generated low scores in the experiment. This difference may be due some attributes being inter-related, more wide-ranging or the sequential nature of establishing an effective screening programme, with guidelines being the first stage of the process.

Key words - cancer, screening, choice experiment 


\section{Introduction}

What makes an effective cancer screening programme? This is a key question for researchers and policy makers aiming to improve cancer screening programmes in Europe. Cancer screening activities in Europe are embedded within wider health systems but their relationships with them varies. Some are organised largely separately, with their own staff and facilities, and others using existing resources, as examinations are undertaken, either opportunistically or within an organised framework, by gynaecologists and other physicians in their own premises. However, whatever the precise arrangements, the opportunity for screening to achieve health gain depends on the interaction of many elements within and beyond the health system, from an accurate register identifying the target population to a means to ensure and monitor follow up, such as linkage to a cancer registry. Consequently, those responsible for cancer screening must look beyond the aspects of the process over which they have direct control.

The EU-TOPIA project (Towards improved screening for breast, cervical and colorectal cancer in all of Europe) aims to optimise screening in Europe ${ }^{1}$. Although the European Council issued comprehensive recommendations for breast, cervical and colorectal cancer screening in Europe, performance indicators show that there is still a need to develop more effective population-based screening programs in Europe ${ }^{2-5}$. As demonstrated in previous studies by the authors ${ }^{6-8}$ there remain many barriers to effective cancer screening within broader health systems and beyond. Defining an effective screening programme is one step in the process towards improvement and overcoming these system barriers.

Given the growing burden of healthcare and the limited resources available, there is a need for researchers and policy makers to prioritise areas for attention. Choice experiments offer a means to identify the importance attributed to different factors affecting healthcare delivery whilst taking account of the real life trade-offs that need to be made in an environment of limited resources. Previous experiments of this type of methodology have been used to assess patient preferences for cancer screening, thereby informing the design and operation of services ${ }^{9,10}$. This focus reflects the preponderance, in the literature on barriers to effective screening, of barriers to individual participation rather than service or system level barriers (a systematic review by the authors has been submitted for publication elsewhere). This experiment aims to readdress the balance of evidence by investigating the attitudes and beliefs of those responsible for the organisation of screening programmes at a national and regional level. 


\section{Methods}

We used a conjoint analysis methodology incorporating a single ranking exercise. Our methodology was informed by a review of the available literature ${ }^{11-14}$, a systematic literature review of discrete choice methods ${ }^{15}$ and insights gained from prior studies conducted by the authors $^{16-18}$.

As shown in Figure 1, the conjoint methodology included four key stages. The first stage is to identify a list of key attributes that we believe, based on the available evidence, to be essential for an effective screening programme. For example, as will be described later, one attribute of a screening programme is that it uses a register that includes the entire population. Each attribute has a series of levels. Using the same example, we selected only two possible levels, yes, such a register is used, or no, none is used. It would be possible to have had more levels, for example with three levels, a register that covered $100 \%, 70-99 \%$, and less than $70 \%$. However, we had no sound basis for choosing these thresholds. The second stage is to generate profiles of screening programmes, each specifying one level for each attribute. Thus, in the example above, some profiles would include a register covering the entire population and others would not. Each would also contain similar information on all the other attributes. Even with a small number of attributes and levels, the number of possible combinations is enormous, so we use the orthoplan routine in the SPSS software package ${ }^{19}$ to generate a smaller sub-sample of all possible profiles. The resulting profiles have two important characteristics. They are balanced, that each level in each attribute appears the same number of times, and are orthogonal, so that every pair of levels, where one is from one attribute and one from another attribute, appear the same number of times in the design. These characteristics are necessary for the final stage. Before that, in the third stage, individuals are asked to rank all profiles from the best to worst cancer screening programmes. The characteristics of the profiles that have been generated in this way, combined with the ranking by participants, are then combined in the fourth and final stage, using the conjoint routine in SPSS, to calculate weights relating to the importance placed on

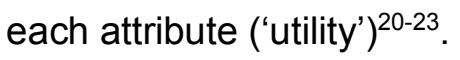

Following the conjoint experiment, we engaged in a discussion of the results with participants in the study in order to gain a better understanding of their meaning and implications.

\section{Establishing the attributes}


Following our literature reviews, we used three key papers as a basis for establishing a list of key attributes and potential levels to use in the conjoint exercise. Firstly, the European Commission report on the implementation of the Council recommendation on cancer screening (reprint May 2017) ${ }^{2}$ identified the following essential stages of a screening programme: information and invitation of the target population; performing the screening test; assessment or follow-up of abnormalities detected; referral for diagnostic confirmation and treatment; and treatment, if applicable. Secondly, Anttila et al. $2015^{24}$ described as essential the following phases of a programme: identification and personal invitation of each eligible individual; performance of the screening test - examination or procedure; diagnostic work-up of people with detected abnormalities; and when indicated, treatment, surveillance and aftercare. Thirdly, Lynge et al. $2012^{25}$ described essential phases of the implementation of a screening programme as : before planning, planning, feasibility, pilot, roll-out, running programme, and sustainability. From these papers, we collated a list of attributes and then prioritised 13 of them based on knowledge obtained from previous work conducted as part of the EU-TOPIA project.

We conducted a pilot with five healthcare researchers in London to assess the feasibility of the required task using the initial list of attributes. We asked the researchers to do a ranking exercise with a set of 27 profiles generated from the 13 attributes and we then discussed their experience in a qualitative manner. The researchers were not experts in cancer screening but this allowed us to ensure the feasibility of the exercise and provide feedback on the meaningfulness of the descriptions.

The qualitative feedback from the pilot indicated that the large number of profiles we initially considered was excessive as it was impractical to rank them in one exercise from best to worst. Consequently, we reduced the numbers of attributes from 13 to 10 and minimised the number of levels to two per attribute (as shown in Table 1) so that respondents had 12 profiles to rank, which was deemed manageable by those involved in the pilot.

The qualitative assessment of the pilot also suggested that a refinement of the attribute descriptions was required to facilitate the exercise. The initial short descriptions were considered to be open to interpretation. We added long descriptions to add clarification (Table 2).

The descriptions of the attributes and levels were then reviewed by a team of experts in cancer screening programmes from six European countries taking part in the EU-TOPIA project to ensure external validity.

\section{Generation of profiles}


We created a data file listing the ten attributes and two alternative levels. We used a standard SPSS conjoint syntax for orthogonal design (orthoplan) ${ }^{19-22}$ to generate a series of profiles combining these attributes and levels, to generate a series of 12 profiles. Listing all combinations would generate a large number of profiles. Thus, the software generated just enough combinations that allow the authors to calculate the weights that participants give to each attribute, based on their rankings. The final result was a matrix of 12 profiles made up of 10 attributes, each with two levels ('yes' or 'no')(Table 3).

\section{Ranking exercise and analysis}

An online form, with a file including the 12 profiles, was created and circulated to participants. The materials included an explanation of the exercise, description of the profiles and table for the participant to complete with their results. Copies of the materials are included in Appendix A and B. We used the conjoint analysis function within SPSS software ${ }^{19-22}$ consistent with previous studies ${ }^{17,18}$.

\section{Qualitative discussion}

A EU-TOPIA project workshop was held in Budapest in September 2017 to which respondents of the survey were invited. During the workshop, a facilitated group discussion of an hour duration was held with a selection of respondents. We explored whether respondents were surprised at the results of the survey, whether they believed that all important attributes had been included, and discussed the respondents' response to the conjoint ranking methodology.

\section{Results}

\section{Sample characteristics}

The sample included 66 participants from 31 countries taking part in the EU-TOPIA workshop. Respondents were mainly from cancer screening organisations, researchers or policy-makers, whose more specific roles are shown in Figure 2 (11 did not specify their role).

\section{Importance scores}

The results of the experiment are shown in Figure 3 and Table 4. There was a noticeable division of results between the four most important attributes, all with an average importance 
factor of over 15 and the other six attributes which had an importance factor of less than seven.

\section{Follow-up workshop with respondents}

Methods such as this, by forcing trade-offs, can reveal preferences that would otherwise remain concealed. In the workshop discussion, participants were reluctant to accept that they had rated quality assurance as of low importance and were surprised at this result. We explored several explanations for the difference between the results of the experiment and the group opinion with them. One explanation was that some of the attributes are sequential. For example, guidelines can only be adhered to once in place and quality can only be assured once other attributes are developed (e.g. monitoring) so those ranking profiles in the experiment may have taken this into account implicitly. Another explanation, although less intuitive, was that guidelines are considered more wide-ranging than quality assurance and therefore ranked higher by respondents aiming to maximise their choice. Knowledge of the attributes in practice may also have influenced ranking in the experiment. For example, the group indicated that actual guidelines are sometimes based on expert opinion rather than higher-level evidence and are infrequently updated. Therefore, this issue should be prioritized as a key factor among others. Knowledge of this may affect how important this factor is in an effective screening programme.

\section{Discussion}

\section{Strengths and limitations of the study}

So far, much of the literature on cancer screening has focused on those elements within the organisational boundaries of organised programmes. Yet, to achieve optimal outcomes, it can be argued that it is necessary to look more widely. This experiment suggests that those involved in the operation of cancer screening agree with our applied concept. We are unaware of a similar exercise being undertaken previously. Based on this experience we also suggest that performance indicators for cancer screening programmes ${ }^{2}$ should take a broader perspective in assessing effectiveness.

The study used an established methodology for conjoint analysis ${ }^{20-22}$. The list of attributes used in the study was formulated in several phases, including data collection (from the literature), data reduction, removing inappropriate attributes and wording (refined as a result of the pilot and reviewed by experts) in line with recommendations in the literature ${ }^{26}$. The trial phase with healthcare researchers and review by experts ensured external validity. In a 
follow-up phase, the results were validated with respondents in a qualitative manner which also allowed a better understanding of the quantitative results. The selected sample included experts on cancer screening programmes throughout Europe, with coverage of 31 countries.

The main limitation of the study was the sample size. Ideally, the sample size would have been larger ${ }^{27}$. However, the addition of a qualitative phase allowed us to use the quantitative results as a source for discussion and future actions. In addition, the sample focused on researchers, organisers of screening programmes and policy makers. A more extensive study might have included service providers, health professionals directly involved in the screening process, and people invited for screening. Previous research has shown that patient preferences, as perceived by healthcare professionals, may differ from those of the patients themselves ${ }^{28}$ so a comprehensive sample including all stakeholders would increase the robustness of any study.

The post-experiment discussion with respondents suggested some disagreement with the results of the experiment. Previous research in cancer screening has also shown differences when comparing the results of choice experiments versus ordinary rating, ranking or survey questions $^{29,30}$, even if overall there was agreement with the attribute ranked as most important ones ${ }^{29}$. However, discussion at the workshop indicated that some attributes were inter-related, sequential or more wide-ranging and this might have affected how people ranked the profiles. For example, without implemented evidence based guidelines, assuring quality would not be feasible. Conversely, assuring quality may make the assumption that guidelines and a screening organisation have already been established. This may explain the low score for the quality assurance attribute.

\section{Comparison with the literature}

As previously mentioned, previous conjoint studies have been conducted with cancer screening programmes but the focus has generally been on understanding patient preferences $^{9,10,31-36}$ or, on occasion, physicians ${ }^{28}$, to inform strategies to increase uptake. For example, discrete choice experiments have investigated patient preferences for the test options made available ${ }^{30,37}$, test intervals ${ }^{37}$, travel time ${ }^{30}$, and financial incentives or copayments ${ }^{30}$ Other studies have also investigated the impact of including labels for specific screening tests versus non-labelling of a list of attributes ${ }^{38}$. In a review of the literature on breast, cervical and colorectal cancer screening ${ }^{9}$, of 22 included studies on patient preferences, 15 assessed test attributes, two studies assessed healthcare delivery, and five studies included both. Test attributes had a significant effect on choice of test and healthcare delivery (how screening is organised) had a mixed effect. This review recommended further 
research on barriers to uptake, particularly research including healthcare delivery, as this study aims to do. Another systematic review of colorectal cancer screening ${ }^{10}$ showed that people preferred screening to non-screening and were willing to trade-off complications to get more clinical benefit. A similar review of 28 cancer treatment preference studies ${ }^{39}$ found that most attributes related to outcomes $(70 \%)$ while process and cost attributes only related to $25 \%$ and $5 \%$ of evidence respectively, showing less attribute importance as well as being less often included in studies.

\section{Implications}

There is a lack of evidence on the priorities of policy makers who are forced to make tradeoffs in cancer screening. Discrete choice methods can be used to evaluate healthcare interventions, services and policies including both outcomes and process attributes ${ }^{40,41}$, including priority setting ${ }^{42}$. As previously mentioned, when looking at patient preferences, factors such as convenience (mode of administration and location of treatment) may overlap with health service design. However, choice experiments can also be used to examine health system and service attributes. In previous studies discrete choice has been used: 1) to assess the views of policymakers on health technology assessment ${ }^{43}, 2$ ) to assess decision making criteria by policy makers in five countries, including efficiency and equity ${ }^{44}$, 3 ) to explore reforms to payment systems ${ }^{45} 4$ ) to assess pharmacy services ${ }^{46}$ and 5) to inform health workforce policy ${ }^{47}$. These studies have demonstrated the value of choice experiments in designing health services. However, none of these studies have addressed cancer screening directly.

\section{Conclusion}

This choice experiment concluded that having up-to-date and evidence-based guidelines was the most important factor in an effective screening programme, followed by systematically monitoring screening uptake, having a population register covering all the eligible population and monitoring long-term outcomes. When required to trade-off, respondents selected these attributes rather than quality assurance or guideline adherence. The follow-up qualitative research on these priorities suggests that quality assurance and guideline adherence are also important to them. However, the main contribution of this exercise was to stimulate a valuable discussion on the role of quality assurance and guidelines that might otherwise not have happened, and which allowed some important issues to emerge. The factors that were deemed most important in the choice experiment may be the most important ones to have in the first place, with quality assurance dependent 
upon them. This suggests that, where resources are limited, establishing evidence-based and up-to-date guidelines should be a priority, followed by monitoring uptake, and ensuring a comprehensive population register before developing other aspects of the screening programme. However, a more comprehensive study, including a wider group of stakeholders and a more extensive list of independent attributes would provide even more additional insights.

\section{References}

1. EU-TOPIA (Towards improved screening for breast, cervical and colorectal cancer in all of Europe). http://eu-topia.org/about-eu-topia/who-we-are/.

2. International Agency for Research on Cancer. Cancer Screening in the European Union (2017) - 2nd Report on the implementation of the Council Recommendation on cancer screening. European Commission;2017.

3. European Colorectal Cancer Screening Guidelines Working G, von Karsa L, Patnick J, et al. European guidelines for quality assurance in colorectal cancer screening and diagnosis: overview and introduction to the full supplement publication. Endoscopy. 2013;45(1):51-59.

4. European Commission. European guidelines for quality assurance in breast cancer screening and diagnosis, 4th edition, supplements. 2013.

5. European Commission. European guidelines for quality assurance in cervical cancer screening - Second edition. 2015.

6. Turnbull E, Priaulx J, van Ravesteyn NT, et al. A health systems approach to identifying barriers to breast cancer screening programmes. Methodology and application in six European countries. Health Policy. 2018.

7. Turnbull E, Priaulx J, de Kok I, et al. Results of a health systems approach to identify barriers to population-based cervical and colorectal cancer screening programmes in six European countries. Health Policy. 2018.

8. Priaulx J, de Koning HJ, de Kok I, Szeles G, McKee M. Identifying the barriers to effective breast, cervical and colorectal cancer screening in thirty one European countries using the Barriers to Effective Screening Tool (BEST). Health Policy. 2018.

9. Mansfield C, Tangka FK, Ekwueme DU, et al. Stated Preference for Cancer Screening: A Systematic Review of the Literature, 1990-2013. Prev Chronic Dis. 2016;13:E27.

10. Wortley S, Wong G, Kieu A, Howard K. Assessing stated preferences for colorectal cancer screening: a critical systematic review of discrete choice experiments. Patient. 2014;7(3):271-282.

11. Lancsar $\mathrm{E}$, Louviere J. Conducting discrete choice experiments to inform healthcare decision making: a user's guide. Pharmacoeconomics. 2008;26(8):661-677.

12. Lancsar E, Louviere J, Donaldson C, Currie G, Burgess L. Best worst discrete choice experiments in health: methods and an application. Soc Sci Med. 2013;76(1):74-82.

13. Hall J, Viney R, Haas M, Louviere J. Using stated preference discrete choice modeling to evaluate health care programs. J Bus Res. 2004;57(9):1026-1032.

14. Reed Johnson F, Lancsar E, Marshall D, et al. Constructing experimental designs for discretechoice experiments: report of the ISPOR Conjoint Analysis Experimental Design Good Research Practices Task Force. Value Health. 2013;16(1):3-13.

15. Ryan $M$, Scott DA, Reeves $C$, et al. Eliciting public preferences for healthcare: a systematic review of techniques. Health Technol Assess. 2001;5(5):1-186.

16. Burton CD, Entwistle VA, Elliott AM, Krucien N, Porteous T, Ryan M. The value of different aspects of person-centred care: a series of discrete choice experiments in people with longterm conditions. BMJ Open. 2017;7(4):e015689. 
17. O'Hara NN, Slobogean GP, Mohammadi T, et al. Are patients willing to pay for total shoulder arthroplasty? Evidence from a discrete choice experiment. Can J Surg. 2016;59(2):107-112.

18. Sassi F, McKee M. Do clinicians always maximize patient outcomes? A conjoint analysis of preferences for carotid artery testing. J Health Serv Res Policy. 2008;13(2):61-66.

19. IBM. IBM SPSS Software. https://www.ibm.com/analytics/us/en/technology/spss/. Accessed 31 October, 2017.

20. $\quad$ SPSS Inc. SPSS Conjoint TM 8.0. Chicago, USA1997.

21. IBM. IBM SPSS Conjoint 22. http://www.sussex.ac.uk/its/pdfs/SPSS Conjoint 22.pdf. Accessed 31 October, 2017.

22. Vienna University of Economics and Business. How to analyse SPSS conjoint. Applied statistics using SPSS http://statmath.wu-wien.ac.at/courses/as spss/Conjoint.

23. Hauber AB, Gonzalez JM, Groothuis-Oudshoorn CG, et al. Statistical Methods for the Analysis of Discrete Choice Experiments: A Report of the ISPOR Conjoint Analysis Good Research Practices Task Force. Value Health. 2016;19(4):300-315.

24. Anttila A, Lonnberg S, Ponti A, et al. Towards better implementation of cancer screening in Europe through improved monitoring and evaluation and greater engagement of cancer registries. European journal of cancer. 2015;51(2):241-251.

25. Lynge E, Tornberg S, von Karsa L, Segnan N, van Delden JJ. Determinants of successful implementation of population-based cancer screening programmes. European journal of cancer. 2012;48(5):743-748.

26. Helter TM, Boehler CE. Developing attributes for discrete choice experiments in health: a systematic literature review and case study of alcohol misuse interventions. J Subst Use. 2016;21(6):662-668.

27. de Bekker-Grob EW, Donkers B, Jonker MF, Stolk EA. Sample Size Requirements for DiscreteChoice Experiments in Healthcare: a Practical Guide. Patient. 2015;8(5):373-384.

28. Marshall DA, Johnson FR, Kulin NA, et al. How do physician assessments of patient preferences for colorectal cancer screening tests differ from actual preferences? A comparison in Canada and the United States using a stated-choice survey. Health Econ. 2009;18(12):1420-1439.

29. Pignone MP, Brenner AT, Hawley S, et al. Conjoint analysis versus rating and ranking for values elicitation and clarification in colorectal cancer screening. J Gen Intern Med. 2012;27(1):45-50.

30. Pignone MP, Crutchfield TM, Brown PM, et al. Using a discrete choice experiment to inform the design of programs to promote colon cancer screening for vulnerable populations in North Carolina. BMC Health Serv Res. 2014;14:611.

31. de Bekker-Grob EW, Rose JM, Donkers B, Essink-Bot ML, Bangma CH, Steyerberg EW. Men's preferences for prostate cancer screening: a discrete choice experiment. Br J Cancer. 2013;108(3):533-541.

32. Howard K, Salkeld GP, Patel MI, Mann GJ, Pignone MP. Men's preferences and trade-offs for prostate cancer screening: a discrete choice experiment. Health Expect. 2015;18(6):31233135.

33. Marshall DA, Johnson FR, Phillips KA, Marshall JK, Thabane L, Kulin NA. Measuring patient preferences for colorectal cancer screening using a choice-format survey. Value Health. 2007;10(5):415-430.

34. Phillips KA, Van Bebber S, Marshall D, Walsh J, Thabane L. A review of studies examining stated preferences for cancer screening. Prev Chronic Dis. 2006;3(3):A75.

35. van Dam L, Hol L, de Bekker-Grob EW, et al. What determines individuals' preferences for colorectal cancer screening programmes? A discrete choice experiment. European journal of cancer. 2010;46(1):150-159. 
36. Wordsworth S, Ryan M, Skatun D, Waugh N. Women's preferences for cervical cancer screening: a study using a discrete choice experiment. Int J Technol Assess Health Care. 2006;22(3):344-350.

37. Hol L, de Bekker-Grob EW, van Dam L, et al. Preferences for colorectal cancer screening strategies: a discrete choice experiment. Br J Cancer. 2010;102(6):972-980.

38. de Bekker-Grob EW, Hol L, Donkers B, et al. Labeled versus unlabeled discrete choice experiments in health economics: an application to colorectal cancer screening. Value Health. 2010;13(2):315-323.

39. Bien DR, Danner M, Vennedey V, Civello D, Evers SM, Hiligsmann M. Patients' Preferences for Outcome, Process and Cost Attributes in Cancer Treatment: A Systematic Review of Discrete Choice Experiments. Patient. 2017.

40. Ryan M, Gerard K. Using discrete choice experiments to value health care programmes: current practice and future research reflections. Appl Health Econ Health Policy. 2003;2(1):55-64.

41. Ryan M. Discrete choice experiments in health care. BMJ. 2004;328(7436):360-361.

42. Farrar S, Ryan M, Ross D, Ludbrook A. Using discrete choice modelling in priority setting: an application to clinical service developments. Soc Sci Med. 2000;50(1):63-75.

43. Koopmanschap MA, Stolk EA, Koolman X. Dear policy maker: have you made up your mind? A discrete choice experiment among policy makers and other health professionals. Int J Technol Assess Health Care. 2010;26(2):198-204.

44. Mirelman A, Mentzakis E, Kinter E, et al. Decision-making criteria among national policymakers in five countries: a discrete choice experiment eliciting relative preferences for equity and efficiency. Value Health. 2012;15(3):534-539.

45. Kessels R, Van Herck P, Dancet E, Annemans L, Sermeus W. How to reform western care payment systems according to physicians, policy makers, healthcare executives and researchers: a discrete choice experiment. BMC Health Serv Res. 2015;15:191.

46. Vass $C$, Gray E, Payne K. Discrete choice experiments of pharmacy services: a systematic review. Int J Clin Pharm. 2016;38(3):620-630.

47. Mandeville KL, Lagarde M, Hanson K. The use of discrete choice experiments to inform health workforce policy: a systematic review. BMC Health Serv Res. 2014;14:367.

\section{List of attached table legends}

Table 1: Short description of attributes

Table 2: Long description of attributes (added after pilot phase)

Table 3: Profile matrix of attributes and levels generated by statistical software

Table 4: Detailed breakdown of utility estimates and importance factors $(n=66)$

\section{List of attached figure legends}

Figure 1: A summary of the conjoint analysis methodology

Figure 2: The role of respondents included in the sample $(n=66)$

Figure 3: Attributes ranked most highly by participants $(n=66)$. 


\section{Appendices}

Appendix A - Online conjoint survey form

Appendix B - Conjoint profiles sent to participants 